\title{
Numerical method and calculation of two-phase swirling flows with rigid particles for technical applications
}

\author{
Vadim Akhmetov", Valentina Akhmetova \\ Moscow state university of civil engineering, Yaroslavskoye shosse, 26, Moscow, Russia, 129337
}

\begin{abstract}
Swirling flow with particle deposition effects at the lateral surface is numerically investigated. The flow field calculation results have been obtained as the solutions of the Navier-Stokes equations. Various flow regimes with the formation of axial recirculation zones are presented. The convection-diffusion model is used for the determination of the flow particle concentration and the formation of typical sedimentation zones.
\end{abstract}

\section{Introduction}

Swirling flows have many applications in industrial engineering, in particular, in hydrotechical construction, in gas turbine combustion chambers and in vortex chambers, for the intensification of heat and mass transfer processes [1-5]. The stability of the swirling axisymmetric flows is considered in [6-7]. In this work, a vortex atomizer model which generates aerosol particle flows is discussed. The investigated device is modeled as a vertical pipe with a tangential swirler at the bottom. It is placed at some distance from the horizontal plane with the input powder. The airflow is moved through the tangential swirler into the pipe by creating a pressure gradient at the top. The powder is inducted into the pipe through the bottom section. After the intermixing of the two-phase blend, an aerosol flow at the top of the pipe is formed.

\section{Formulation of the problem and numerical procedure}

The present analysis is based upon the numerical solution of the full Navier-Stokes equations for laminar axisymmetric viscous flow. In the cylindrical coordinate system $r, \varphi, z$ the Navier-Stokes equation can be represented in terms of the stream function $\psi$, the vorticity $\Omega$ and azimuthal velocity $V_{z}$ in form

$$
\frac{1}{r} \frac{\partial^{2} \psi}{\partial z^{2}}+\frac{\partial}{\partial r}\left(\frac{1}{r} \frac{\partial \psi}{\partial r}\right)=-\Omega
$$

* Corresponding author: AhmetovVK@mgsu.ru 


$$
\begin{gathered}
\frac{\partial \Omega}{\partial t}+\frac{\partial}{\partial z}\left(V_{z} \Omega\right)+\frac{\partial}{\partial r}\left(V_{r} \Omega\right)=\frac{1}{\operatorname{Re}}\left[\frac{\partial^{2} \Omega}{\partial z^{2}}+\frac{\partial^{2} \Omega}{\partial r^{2}}+\frac{\partial}{\partial r}\left(\frac{\Omega}{r}\right)\right]+G^{2} \frac{1}{r} \frac{\partial\left(V_{\varphi}\right)^{2}}{\partial z} \\
\frac{\partial V_{\varphi}}{\partial t}+\frac{\partial}{\partial z}\left(V_{z} V_{\varphi}\right)+\frac{1}{r} \frac{\partial}{\partial r}\left(r V_{r} V_{\varphi}\right)+\frac{V_{r} V_{\varphi}}{r}=\frac{1}{\operatorname{Re}}\left[\frac{\partial^{2} V_{\varphi}}{\partial z^{2}}+\frac{1}{r} \frac{\partial}{\partial r}\left(r \frac{\partial V_{\varphi}}{\partial r}\right)-\frac{V_{\varphi}}{r^{2}}\right] \\
V_{r}=-\frac{1}{r} \frac{\partial \psi}{\partial z}, V_{z}=\frac{1}{r} \frac{\partial \psi}{\partial r}, \Omega=\frac{\partial V_{r}}{\partial z}-\frac{\partial V_{z}}{\partial r}
\end{gathered}
$$

The system of equation (1)-(4) is written in the conservative form and contains two dimensionless parameters: the Reynolds number $\operatorname{Re}=U R / v$, where $v$ is the kinematic viscosity, and the swirl ratio $G=W_{0} / U$.

Let $z_{1}$ be the distance from the pipe to the plane with input powder. The flow enters the pipe throughout the opened lateral part, $0 \leq z \leq z_{1}$, with the radial velocity $V_{0}$ and the uniformly perforated pipe surface, $z_{1} \leq z \leq z_{0}$, with the radial velocity $k V_{0}$ and the azimuthal velocity $W_{0}$. The pore coefficient $k$ is the ratio of open to total area for the lateral surface $z_{1} \leq z \leq z_{0}$. The value $V_{0}$ is defined as

$$
V_{0}=\frac{R U}{2} \frac{1}{\left(z_{0}-z_{1}\right) k+z_{1}}
$$

where $U$ is the given mean flow velocity at the outlet section $z_{k}$.

The flow is considered in the cylindrical domain $D \quad\left(0 \leq z \leq z_{k}, 0 \leq r \leq 1\right)$. The boundary conditions involve specifying the velocity profiles at the inlet section $r=1$, $0 \leq z \leq z_{0}$, no-slip conditions on the rigid surfaces, and symmetry conditions on the axis $r=0$. Soft boundary conditions are imposed in the outlet section $z=z_{k}$. The set of boundary conditions can be written in form

$$
\begin{gathered}
\psi=0, V_{\varphi}=0, \frac{\partial \psi}{\partial z}=0, \quad 0 \leq r \leq 1, \quad z=0 \\
\psi=f_{1}(z), \quad V_{\varphi}=0, \frac{\partial \psi}{\partial r}=0, \quad 0 \leq z \leq z_{1}, \quad r=1 \\
\psi=f_{2}(z), \quad V_{\varphi}=1, \quad \frac{\partial \psi}{\partial r}=0, \quad z_{1} \leq z \leq z_{0}, \quad r=1, \\
\psi=\text { const }, V_{\varphi}=0, \frac{\partial \psi}{\partial r}=0, \quad z_{0} \leq z \leq z_{k}, \quad r=1 \\
\psi=0, \quad V_{\varphi}=0, \quad \Omega=0, \quad 0 \leq z \leq z_{k}, \quad r=0 \\
\frac{\partial \psi}{\partial z}=\frac{\partial \Omega}{\partial z}=\frac{\partial V_{\varphi}}{\partial z}=0, \quad 0 \leq r \leq 1, \quad z=z_{k}
\end{gathered}
$$


To solve numerically the boundary value problem (1)-(4), (6)-(11) the finite difference method was used. The solution of the Poisson equation (1) was obtained using the incomplete reduction method, and the transport equations (2)-(3) were solved using the implicit block iteration method. The diffuse terms were approximated by means of central differences. For approximating the convective terms we used a modified Leonard scheme with quadratic upstream terms of third-order accuracy. Initially, for each time step equation (1) was solved with respect to $\psi$, then the values of $V_{z}$ and $V_{r}$ were calculated from formulas (4) and equation (3) was solved to determine $V_{\varphi}$.

The pressure distribution $p$ was found by taking the calculated velocity flow field into account. The Poisson equation with respect to the pressure gradient has the form

$$
\frac{\partial^{2} p}{\partial z^{2}}+\frac{\partial^{2} p}{\partial r^{2}}+\frac{1}{r} \frac{\partial p}{\partial z}=2\left(-\frac{\partial V_{z}}{\partial r} \frac{\partial V_{r}}{\partial z}+\frac{\partial V_{r}}{\partial r} \frac{\partial V_{z}}{\partial z}+G^{2} \frac{V_{\varphi}}{r} \frac{\partial V_{\varphi}}{\partial r}\right)
$$

The boundary conditions for equation (12) are obtained from the equations of motion and have the form

$$
\begin{gathered}
\frac{\partial p}{\partial z}=-\frac{1}{\operatorname{Re}}\left(\frac{\partial \Omega}{\partial r}+\frac{\Omega}{r}\right), 0 \leq r \leq 1, \quad z=0, \quad z=z_{k} \\
\frac{\partial p}{\partial r}=-\frac{1}{\operatorname{Re}} \frac{\partial \Omega}{\partial z}+\frac{V_{\varphi}^{2}}{r}+\frac{V_{r}^{2}}{r}, 0 \leq z \leq z_{k}, \quad r=1 \\
\frac{\partial p}{\partial r}=0, \quad 0 \leq z \leq z_{k}, \quad r=0
\end{gathered}
$$

For solving the boundary-value problem (12)-(15) the implicit block iteration method was used.

\section{Flow field calculation results}

The flow fields corresponding to the boundary-value problem (1)-(11) were calculated for $\operatorname{Re}=100-2000, G=0-8, k=0.4-0.9, \quad z_{0}=1.4-2.4$, and $z_{1}=0.4-1.4$. The results show that when a critical value of swirl is reached, a recirculation zone is formed.

The typical streamline patterns for the calculated flows are presented in Fig. 1. Consider the flow field structure for an increase of swirl ratio $G$ and fixed parameters $\operatorname{Re}=100$, $k=0.5, z_{0}=2, z_{1}=1$. When the swirl ratio is small $G=1$, the flow pattern is qualitatively similar to that produced by radial blowing in a pipe through the lateral surface. In this case the swirl does not significantly influence the flow and at the outlet section, $\left(z=z_{k}\right)$, a Poiseuille profile is established. With an increase in the swirl, $G=3$ (Fig. 1,a), a reverse flow zone is generated near the wall and the typical looping bend of the streamline is formed. With a further increase in the swirl to $G=6$, the center of this recirculation zone is moved to the plane $z=0$ and the length of the reverse flow is extended both in the axial and radial directions. The maximum value of the azimuthal velocity reaches $-0.5 \mathrm{U}$ and $-0.8 U$ for $G=3,6$ respectively and occurs near the wall.

As the Reynolds number increases, the flow structure changes. For small swirl numbers $(G=2)$ the flow structure and recirculation zone size do not change as $\operatorname{Re}$ is increase to 1000 . However with an increase in swirl to $G=4$ and $\operatorname{Re}=500$ (Fig. 1,b), the shape of the reverse flow region is changed: the recirculation zone is U-shaped and the 


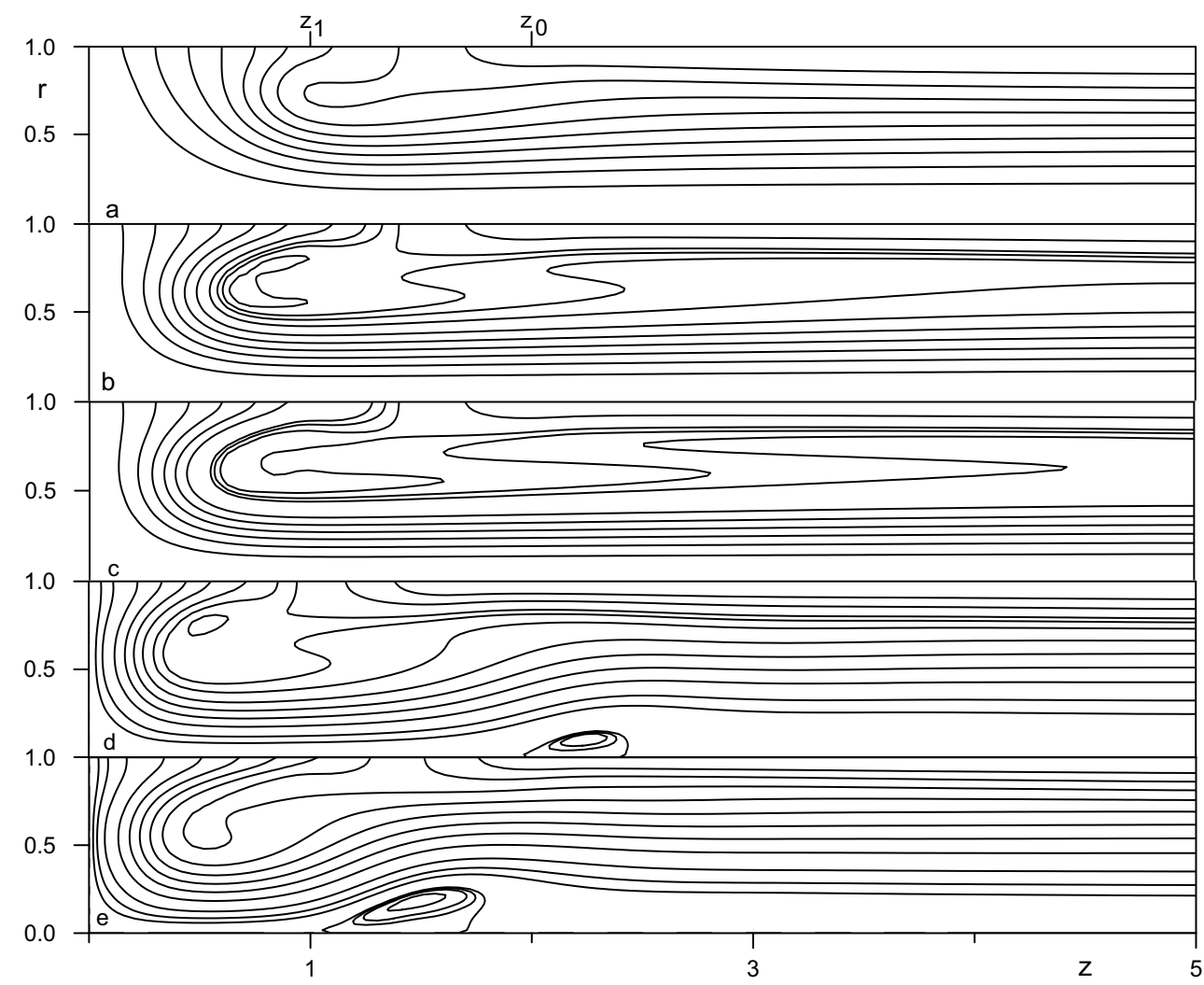

Fig. 1. Streamline patterns: (a) $-\operatorname{Re}=100, G=3, k=0.5, z_{1}=1$; (b) $-\operatorname{Re}=500, G=4, k=0.5, z_{1}=1$; (c) $-\operatorname{Re}=750, G=4, k=0.5, z_{1}=1$; (d) $-\operatorname{Re}=250, G=4, k=0.5, z_{1}=0.6$; (e) $-\operatorname{Re}=250, G=6$, $k=0.7, z_{1}=1$.

streamlines behind it make a bend. At higher Reynolds numbers the length of the reverse flow zone increases and extends to the outlet section, $z=z_{k}$, for $\operatorname{Re}=750$ (Fig. 1,c).

When the pipe is moved to the plane $z=0$, the flow rate entering the pipe through the opened lateral part without the swirl is decreased. Subsequent changes in flow field structure are similar to those expected for an increase in swirl ratio, $G$. Thus for small Reynolds number and swirl ( $\operatorname{Re}=100, G=3$ ) with decrease $z_{1}=0.8,0.6$ the recirculation zone is moved to the plane $z=0$, but the flow patterns are not changed qualitatively.

At higher Reynolds number and swirl ratio $(\operatorname{Re}=250, G=4)$ a decrease of the distance $z_{1}$ leads to an appearance of zigzag streamlines at $z_{1}=0.8$ and then at $z_{1}=0.6$ (Fig. 1,d). The recirculation zone is formed near the axis. There is analogous to the vortex breakdown phenomenon [8-10].

Increasing the pore coefficient $k$ for other fixed parameters leads to an increase of the flow rate entering the pipe with the swirl. As in the previous case there is no significant change to flow field structure for $k$ from 0.5 to 0.9 , small Reynolds number $\operatorname{Re}=100$, 250 , and medium values of $G=2$ at $z_{0}=2, z_{1}=1$. With further increase in the swirl to $G=6$ at $\operatorname{Re}=250$, and $k=0.6$ the typical looping bend of the streamline is formed. With an increase in $k$ to 0.7 a reverse flow zone with a toroidal structure appears near the axis which moves upstream to $k=0.8$ (Fig. 1,e). 


\section{Convection-diffusion model}

The numerical investigations of two-phase flow with rigid particles are based upon the diffusion model. We assume that the influence of the particle motion on the basic flow is negligible. The flow field is defined as a sum of the particle velocity and the liquid phase velocity. In this case the equation of volume global continuity transforms to the diffusion equation for the particle concentration

$$
\frac{\partial c}{\partial t}+\frac{\partial}{\partial z}\left(V_{z} c\right)+\frac{1}{r} \frac{\partial}{\partial r}\left(r V_{s} c\right)=\frac{1}{\operatorname{Re} S c}\left[\frac{\partial^{2} c}{\partial z^{2}}+\frac{1}{r} \frac{\partial}{\partial r}\left(r \frac{\partial c}{\partial r}\right)\right]
$$

in which $c$ is the particle concentration, $S c=v / D$ is the Schmidt number, and $D$ is the diffusion coefficient. The convection-diffusion equation (16) has been used for investigations of particle deposition in [11-13].

For the determination of $V_{s}$ in this case experimental results [14] have been used. It was found, that for the particle motion in a vortex chamber only two forces are important: the centrifugal force $F_{c}$ and the Stokes force $F_{s t}$ :

$$
F_{c}=\frac{4}{3} \pi r_{p}^{3} \rho_{p} \frac{V_{\varphi}^{\prime 2}}{r}, F_{s t}=6 \pi \mu r_{p}\left(V_{s}^{\prime}-V_{r}^{\prime}\right)
$$

where the prime denotes dimensional velocities.

We require that the Taylor number $\mathrm{Ta}$ meet the following condition:

$$
T a=\frac{W_{0} r}{v}<<1
$$

Then it is permissible to assume that the azimuthal velocity of the particle is equal to the azimuthal velocity of the liquid phase. Hence, the deposition velocity $V_{S}$ can be found from the particle motion equation in the radial direction:

$$
\frac{d V_{s}}{d t}=G^{2} \frac{V_{\varphi}^{2}}{r}-\frac{1}{2 S t}\left(V_{s}-V_{r}\right), \quad S t=\frac{\rho_{p} r_{p}^{2} U}{9 v \rho R}
$$

The velocity $V_{s}(z)$ is defined from (19) as an average value with respect to $r$, taking into consideration that $S t<<1$ and $G^{2} \cdot S t=O(1)$ :

$$
V_{S}(z)=2 S t G^{2}\left\langle V_{\varphi}^{2} / r\right\rangle+\left\langle V_{r}\right\rangle
$$

The obtained distribution $V_{S}(z)$ is substituted into equation (16).

Let us assume that the input powder location is the domain $P\left(0 \leq r \leq r_{1}, z=0\right)$. Therefore the boundary conditions for equation (16) can be written:

$$
\begin{gathered}
z=0: \quad c=1, \quad 0 \leq r \leq r_{1} ; \quad \frac{\partial c}{\partial z}=0, r_{1} \leq r \leq 1 \\
z=z_{k}: \quad \frac{\partial c}{\partial z}=0, \quad 0 \leq r \leq 1
\end{gathered}
$$




$$
r=0, r=1: \quad \frac{\partial c}{\partial r}=0, \quad 0 \leq z \leq z_{k}
$$

In this case reflection effects for particle deposition at the lateral pipe surface are not considered. We assume that the particle flux defined as $j=D \partial c / \partial r+V_{s} c$ has only a convection part and the influence of diffusion is negligible $(\partial c / \partial r=0)$.

For solving the boundary value problem (16), (21)-(23) we used a finite difference relaxation method.

\section{Calculation results for flow fields concentration}

Numerical solutions of the boundary value problem (16), (21)-(23) were obtained for $S c=1-10, S t=10^{-5}-10^{-1}$.

First consider the particle deposition process on the lateral pipe surface. The particle concentration flux through the wall is given as $Q_{w}=V_{s} c$. Fig. 2 shows the variation $Q_{w}$ with $z$ for $\operatorname{Re}=100, G=4,4.5,5,6$ (curves 1-4). For $G=4.5$ (curve 2) the distribution has a minimum value at $z \approx 1.95$. The more strongly expressed maximum is noted for $G=5,6$ (curves 3,4 ). At the same time with decreases in the swirl, $G=4$ (curve 1), the deposition profile is practically uniform. Thus a zone with maximum particle deposition is formed. The presence of such a zone is connected with the swirl ratio.

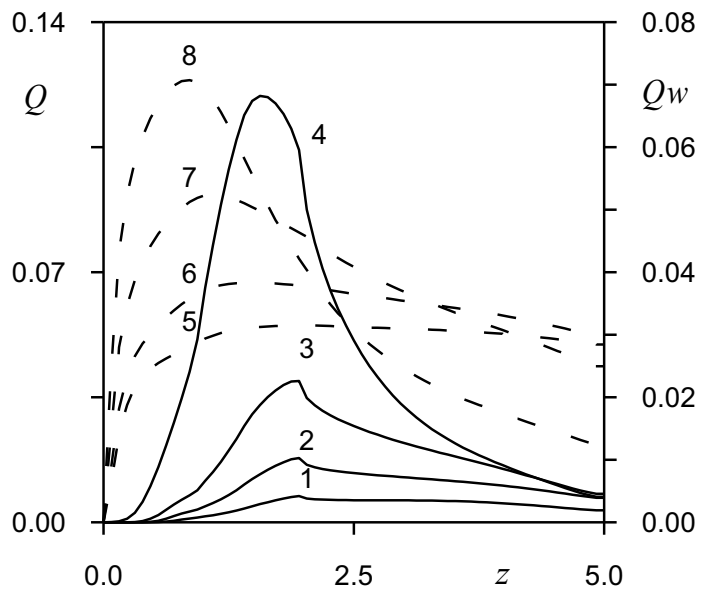

Fig. 2. Flux concentration $Q_{w}(1-4: \mathrm{G}=4,4.5,5,6)$ and flow particle rate $Q(5-8: \mathrm{G}=3,4,5,6)$ for $\mathrm{Re}=100$.

Significant particle deposition on the lateral pipe surface is an undesirable effect. The purpose of the considered device is to form an aerosol flow at the outlet section. Excessive swirl can lead to undesirable effects. This fact is illustrated in Fig. 2. Here the variation of the flow rate $Q(z)$ is given by

$$
Q=2 \pi \int_{0}^{1} V_{z} r c d r
$$




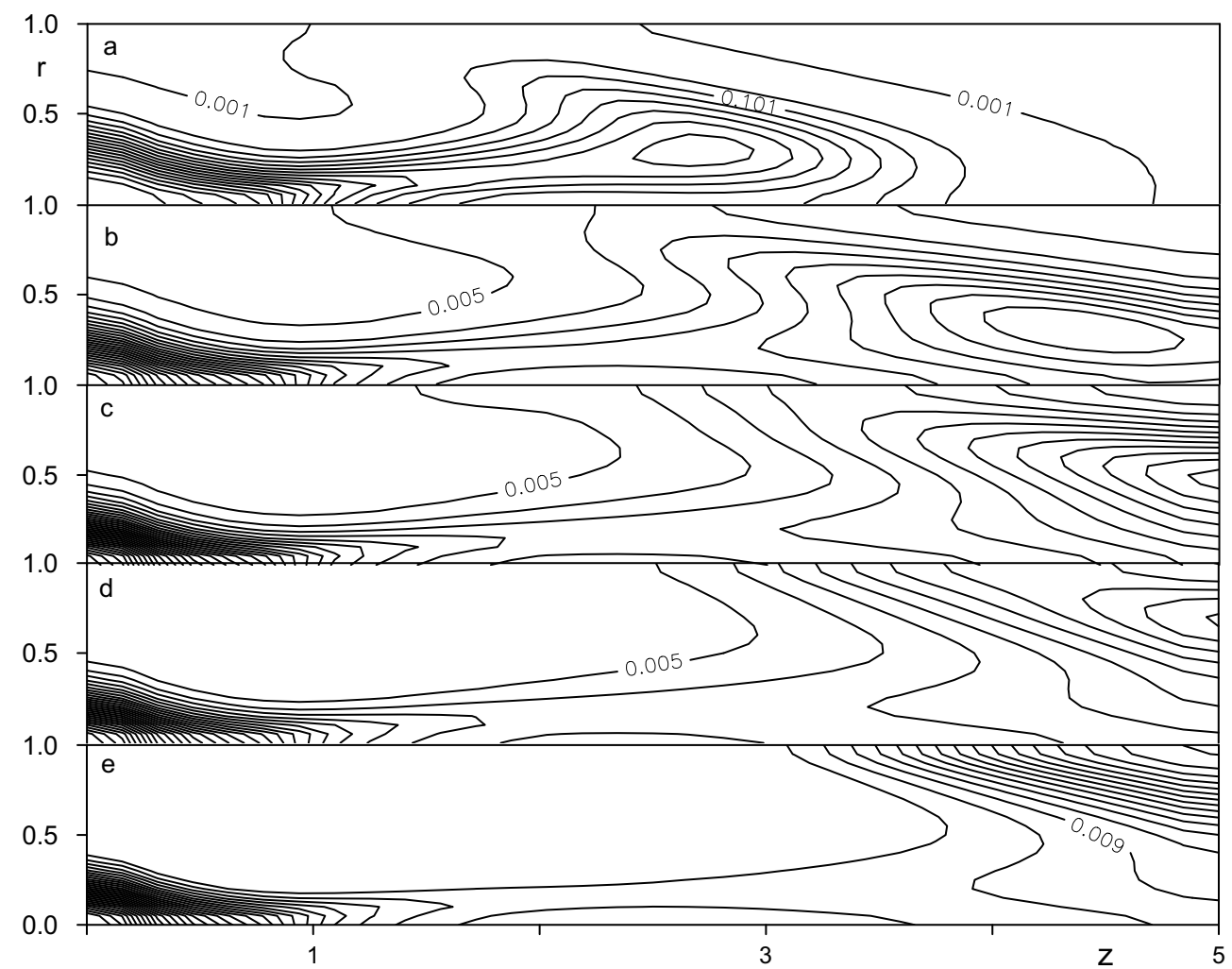

Fig. 3. Concentration fields, $\operatorname{Re}=100, G=3$, (a) - (e): $\mathrm{t}=1,2,3,4,5, \Delta c=0.05,0.025,0.05,0.01$, 0.002 .

for $\mathrm{Re}=100, S c=1, S t=0.022, k=0.5, z_{1}=1, G=3,4,5,6$ (curves 5-8). Fig. 2 shows that small swirl ( $G=3$ ) is insufficient for effective mixing. At the same time, strong swirl ( $G=6$ ) leads to significant particle deposition on the wall. The optimal swirl for the given parameters is $G=5$.

Consider the nonstationary problem for particle transport and deposition. We assume that initially the particles occupy the volume $0 \leq r \leq r_{1}, 0 \leq z \leq z_{1}$. So we specify the initial particle concentration as follows:

$$
c=1,0 \leq r \leq r_{1}, 0 \leq z \leq z_{1} ; \quad c=0, r>r_{1}, z>z_{1}
$$

The set of boundary conditions can be written:

$$
\begin{aligned}
& \frac{\partial c}{\partial z}=0, \quad 0 \leq r \leq 1, \quad z=0, \quad z=z_{k} \\
& \frac{\partial c}{\partial r}=0, \quad 0 \leq z \leq z_{k}, \quad r=0, \quad r=1
\end{aligned}
$$


The solution of the value boundary problem (16), (25)-(27) was carried out for the following parameters: $S c=1, S t=0.022, k=0.5, z_{1}=1, z_{0}=2, r_{1}=0.5$. The typical concentration field for the different time with the steps $(\Delta c)$ is presented in Fig. 3.

\section{Conclusions}

The above examples give a fairly detailed picture of the atomization of a powder to obtain an aerosol using an open cylindrical channel in which a swirling flow is formed. The mathematical model used makes it possible qualitatively to describe the basic properties of the flow, namely, the formation of recirculation zones, the onset of rarefaction in the neighborhood of the flow axis under the action of the swirl, and the entrainment of powder by the flow with partial deposition on the lateral surface of the dispersing apparatus.

\section{References}

1. A. Gupta, G. Lilley, Swirl Flows (Abacus Press, 1984)

2. S.V. Alekseenko, P.A. Kuibin, V.L. Okulov, Introduction to theory of concentrated vortices (Institute of Computer Science, Moscow, 2005)

3. V.K. Akhmetov, V.Ya. Shkadov, Numerical simulation of viscous vortex flows for applications to technics (ASV Publishing House, Moscow, 2009)

4. V.K. Akhmetov, Structure and hydrodynamic stability of swirling flows with recirculation zones (MGSU, Moscow, 2009)

5. V.K. Akhmetov, V.Ya. Shkadov, V.P. Shkadova, Fluid Dynamics, 41 (4), 504 (2006)

6. V.K. Akhmetov, V.Ya. Shkadov, Vestn. Mosk. Univ., Ser. I (5), 54 (2005)

7. V.K. Akhmetov, V.Ya. Shkadov, Vestn. Mosk. Univ., Ser. I (1), 66 (2003)

8. T. Sarpkaya, Trans. ASME, J. Fluids Eng., 111, 5 (1989)

9. M. Escudier, Progr. Aerosp. Sci., 25, 189 (1988)

10. M.A. Herrada, V.N. Shtern, J.M. López-Herrera, Physics of Fluids, 25, 093604 (2013)

11. R. Tsai, Y.P. Chang, T.Y. Lin, J. Aerosol Sci., 29, 811 (1998)

12. J. Mang, E. Minkov, U. Schaflinger, M. Ungarish, Trans. ASME. J. Fluids Eng., 120, $676(1998)$

13. B.T. Tan, Y.S. Liow, L. Mununga, M.C. Thompson, K. Hourigan, Physics of Fluids, 21, 024104 (2009)

14. S.S. Kutateladze, E.P. Volchkov, V.I. Terekhov, Aerodynamics and heat and mass exchange in bounded vortex flows (Institute of Thermophysics, Novosibirsk, 1987) 